\title{
Undertreatment of Tracheal Carcinoma: Multidisciplinary Audit of Epidemiologic Data
}

\author{
Jimmie Honings, $\mathrm{MD}^{1,2}$, Henning A. Gaissert, $\mathrm{MD}, \mathrm{PhD}^{3}$, Ad F. T. M. Verhagen, $\mathrm{MD}^{4}$, \\ Jos A. A. M. van Dijck, PhD $^{5,2}$, Henricus F. M. van der Heijden, MD, $\mathrm{PhD}^{6}$, Lya van Die, $\mathrm{MD}^{7}$, \\ Johan Bussink, MD, PhD ${ }^{8}$, Johannes H. A. M. Kaanders, MD, $\mathbf{P h D}^{8}$, and Henri A. M. Marres, MD, $\mathbf{P h D}^{1}$ \\ ${ }^{1}$ Department of Oto-Rhino-Laryngology and Head and Neck Surgery, Radboud University Nijmegen Medical Centre, \\ P.O. Box 9101, 6500 HB Nijmegen, The Netherlands; ${ }^{2}$ Comprehensive Cancer Centre East, Nijmegen, The Netherlands; \\ ${ }^{3}$ Division of General Thoracic Surgery, Massachusetts General Hospital and Harvard Medical School, Boston, MA, USA; \\ ${ }^{4}$ Department of Cardiothoracic Surgery, Radboud University Nijmegen Medical Centre, Nijmegen, The Netherlands; \\ ${ }^{5}$ Department of Epidemiology and Biostatistics, Radboud University Nijmegen Medical Centre, Nijmegen, \\ The Netherlands; ${ }^{6}$ Department of Pulmonary Diseases, Radboud University Nijmegen Medical Centre, Nijmegen, \\ The Netherlands; ${ }^{7}$ Department of Radiology, Radboud University Nijmegen Medical Centre, Nijmegen, The Netherlands; \\ ${ }^{8}$ Department of Radiation Oncology, Radboud University Nijmegen Medical Centre, Nijmegen, The Netherlands
}

\begin{abstract}
National epidemiologic data were examined to determine the eligibility for curative therapy in tracheal carcinoma. An expert audit of primary tracheal carcinomas registered from 2000 to 2005 with the Netherlands Cancer Registry (NCR) included blinded patient data and radiographic review to assess diagnosis and resectability. Actual treatment was compared with the opinions of a multidisciplinary panel (Radboud panel) and a second reviewer. Of 101 NCR-registered primary tracheal carcinomas, the Radboud panel diagnosis was metastatic disease or local extension of adjacent tumors in 34. Seventeen cases were excluded for missing data. In 50 cases confirmed by panel and a second reviewer, actual treatment consisted of surgery in 12 (24\%), radiotherapy in 29 (58\%), endobronchial treatment in 6 (12\%), and observation in $3(6 \%)$. Both panel and second reviewer identified 16 additional surgical candidates, a total of $28(56 \%)$ of 50 . Treatment recommendations of panel and second reviewer disagreed in four cases (8\%). One-third of NCR-registered primary tracheal carcinomas were misclassified nontracheal primary tumors involving the trachea. A majority of cases meeting audit criteria for diagnosis and surgical resection was treated with other modalities. Interreviewer disagreement was small. The audit of a national cancer registry suggests that incorrect diagnosis and undertreatment are common in rare airway tumors.
\end{abstract}

(C) The Author(s) 2008. This article is published with open access at Springerlink.com

First Received: 5 August 2008;

Published Online: 27 November 2008

J. Honings, MD

e-mail: j.honings@kno.umcn.nl
Primary malignancies of the trachea are rare and challenging tumors. Surgical resection of the involved tracheal segment is the treatment of choice, and long-term diseasefree survival has been reported after tracheal resection. ${ }^{1-3}$ Clinical series suggest that at least half of all patients with primary tracheal carcinoma are surgical candidates. ${ }^{4,5}$ The actual treatment of tracheal cancer in the Netherlands as reported for the period 1989 to 2002 consisted of resection in only $12 \%$ of cases. ${ }^{6}$ Similar proportions were found in epidemiologic studies from Denmark and Finland. ${ }^{7,8}$ The discrepancy between reported ratios of surgical clinics and these epidemiologic studies may be partly based on patient selection. However, some authors suspected a lack of knowledge and a nihilistic attitude toward the treatment of tracheal malignancies. ${ }^{7,8}$

Previous epidemiologic studies contained database information, but no systematic review of radiographs, to explain the discrepancy between clinical and epidemiologic data. ${ }^{7}$ To determine the proportion of the Dutch cancer registry patients with tracheal cancer whose radiographic findings are consistent with the diagnosis and who are candidates for surgical resection, a nationwide audit of cases was conducted.

\section{MATERIALS AND METHODS}

\section{Patient Selection}

In a 6-year period from 2000 to 2005 , all cases coded for primary tracheal carcinoma, labeled C33.9 according to the 
International Classification of Diseases for Oncology, 3rd Revision (ICD-O-3), and reported to the Netherlands Cancer Registry (NCR), through all nine regional cancer registries, were selected. ${ }^{9}$ The main source of notification is the national archive of pathology reports (Pathologisch Anatomisch Landelijk Geautomatiseerd Archief, PALGA), complemented by data from the national hospital discharge registry with case summaries of all patients. The documentation of registered tumors is completed by reviewers at the regional registries who excerpt patient hospital charts. We received permission for this study from the national supervisory committee of the NCR. A waiver for patient consent was obtained with the provision that the primary care physician would agree to enrollment onto the study.

\section{Patient Data Collection}

We acquired the names and addresses of the primary care physicians caring for each patient from the NCR database. All physicians gave permission to include their patients in the study after a written request. Copies of patient charts detailing diagnosis and treatment of tracheal disease were gathered, with additional documentation from referring physicians or specialists providing second opinions. Information on patient and clinical characteristics, diagnostic procedures, tumor pathology, and treatment were entered into a study database. The interval from first seeking care from a specialist to histological diagnosis was obtained and termed "diagnostic delay." Patient charts were searched for documentation of therapeutic decisions and potential contraindications to surgical treatment. Thoracic or otolaryngologic consultation, where documented, was recorded. To protect confidentiality, data entered the audit anonymized, and panel members or second reviewers had no access to patient records or identifiers.

The minimum diagnostic evaluation that was considered complete consisted of cross-sectional imaging by computed tomography (CT) or magnetic resonance imaging and bronchoscopy. Cases were excluded if documentation of evaluation was unavailable or if abnormal findings of either diagnostic modality were not explained or evaluated. Diagnostic images were obtained from radiological hospital archives for each case. From these images, tumor length and extension to other organs, lymph node enlargement, presence of metastatic disease, and evidence of potential comorbid conditions were recorded. Where radiological tumor length diverged from bronchoscopic descriptions, the longer length was recorded. Evaluation of liver and adrenal glands was assumed when a dedicated abdominal CT was obtained or when both organs were shown on the chest study.

\section{Audit Case Review}

Cases were reviewed by a multidisciplinary panel of physicians treating tracheal cancer at Radboud University Nijmegen Medical Centre, Nijmegen, the Netherlands (Radboud panel). The panel consisted of one pulmonary physician (H.H.), one cardiothoracic surgeon (A.V.), one radiologist (L.D.), two radiation oncologists (J.K. and J.B.), and one surgical head and neck oncologist (H.M.). A second review, independent from the Radboud panel, was provided by a thoracic surgeon with experience in tracheal carcinoma (H.G.).

For each case, clinical summaries in combination with CT or magnetic resonance imaging of the trachea and chest were reviewed. Auditors were provided demographic and historic information including sex, age, comorbidity, prior malignancy and treatment, complaints, and excerpts of available radiographic, endoscopic, pathology, or staging reports. The team was blinded to the actual treatment.

\section{Audit of the Diagnosis of Primary Tracheal Carcinoma}

The audit consisted of two parts, both performed by the Radboud panel and a second reviewer. The first part reviewed the primary diagnosis. In the presence of enlarged mediastinal lymph nodes, the tumor was assumed to be primary tracheal carcinoma only when the main tumor mass was located in the trachea, the tracheal tumor was separate from mediastinal lymph nodes, and no other radiographic finding indicated the presence of a primary carcinoma of the lung elsewhere. If the main tumor mass was centered either in a main stem bronchus or in a different organ adjacent to the trachea, in the esophagus, or in the larynx, the tumor was assumed to be a local extension and not primary tracheal carcinoma.

\section{Audit of Treatment}

For the second part of the audit, the Radboud panel and a second reviewer determined resectability and therapeutic options in each case. Tumors were assumed to be resectable when the tumor-bearing tracheal segment could be removed and reconstructed by primary anastomosis, taking into account age and body habitus of the patient (between $20 \%$ and $50 \%$ of the total tracheal length), absence of vital organ invasion such as the heart or great vessels, and absence of mediastinal lymph node involvement. Previous high-dose $(>50 \mathrm{~Gy}$ ) irradiation to the trachea was also regarded as a contraindication to resection.

Radboud panel findings regarding resectability and advised management were consensus based, replicating everyday multidisciplinary oncology practice. The opinion of the second reviewer was compared with the panel 
assessment. The combined opinion of panel and second reviewer was compared with actual treatment, marking cases as surgical candidates only when decided independently by both reviews.

\section{Statistical Analysis}

Statistical testing was performed with SPSS version 14.0 statistical software (SPSS, Chicago, IL). Continuous variables were compared with one-way analysis of variance, and categorical variables were compared with the $\chi^{2}$ test.

\section{RESULTS}

\section{Diagnosis of Tracheal Cancer}

The results from the audit process are depicted in Fig. 1. Excluding two cases in children and two found at autopsy, the NCR identified 104 tracheal carcinomas from 51 hospitals in the period 2000-2005. Three cases for which imaging studies could not be located were excluded from analysis.

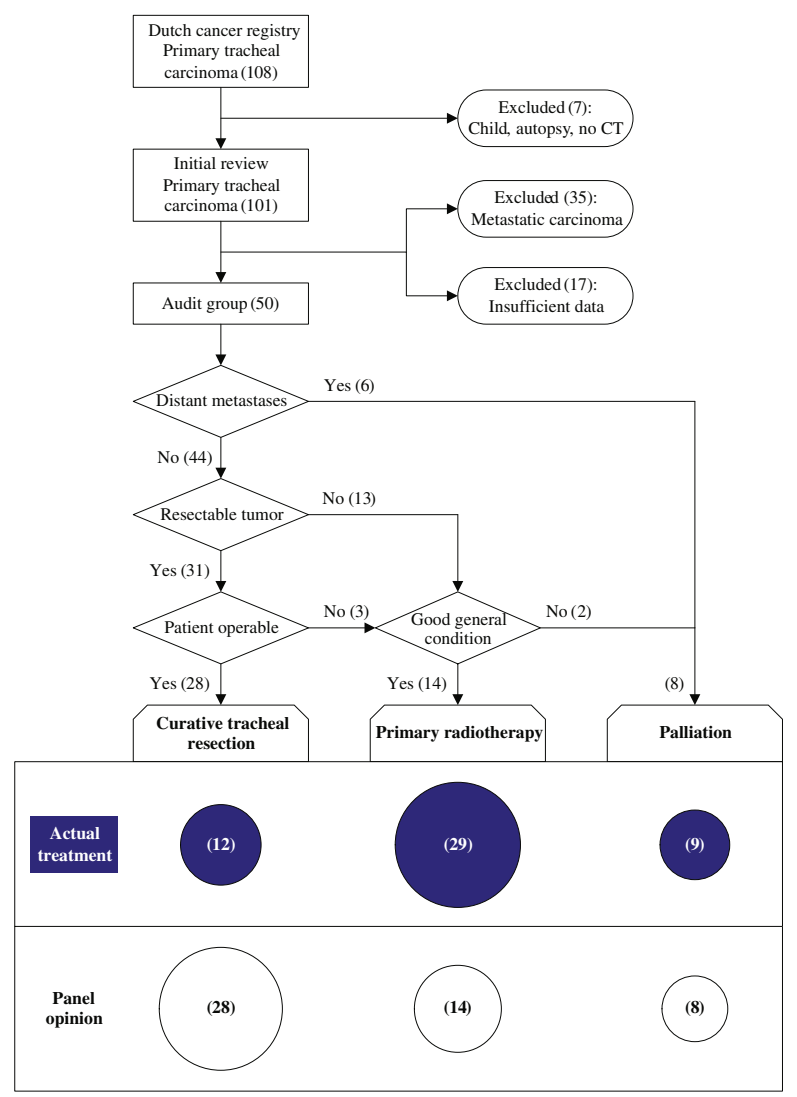

FIG. 1 Depiction of the audit process. Patient is noted as resectable only when indicated by both the review by the Radboud panel and the second reviewer. The actual treatment (blue balloons) is compared with the assessment of the audit (white balloons) in 50 patients with primary tracheal carcinoma $(P=.005)$. Surface area of balloons represents the number of patients in each group
Of 101 cases reviewed in the initial audit, 34 carcinomas (33.7\%) were judged to originate from sites other than the trachea and were thus excluded from further analysis. In 20 (19.8\%) of 101, tracheal biopsy findings positive for carcinoma originated from a primary tumor elsewhere: the lung or main stem bronchus with ingrowth into trachea in 14 cases (squamous cell carcinoma [SCC] in 7, small cell carcinoma in 3, large cell carcinoma in 3, and adenocarcinoma in 1), the esophagus in three (all SCC), and the larynx in three (SCC in two and carcinoma-in-situ in one). One of these laryngeal tumors had been surgically resected.

In the other $14(13.9 \%)$ of 101 cases, mediastinal lymphadenopathy extended into the tracheal lumen or caused malignant stricture without detectable primary tumor. These cases were regarded as a metastatic malignant stricture.

\section{Missing Information}

Seventeen cases, none resected, were excluded as a result of insufficient clinical information. There was no staging for distant metastasis in seven cases; in three, the chest $\mathrm{CT}$ was incomplete, and in four, potential metastasis identified by $\mathrm{CT}$ was not evaluated.

Resectability could not be determined in six cases. Extent and length of an adenoid cystic carcinoma (ACC) was unclear in two, and in the other cases, bronchoscopy was not performed or was not documented, the extent of esophageal invasion was not evaluated, or enlarged mediastinal lymph nodes did not undergo biopsy.

Operability was uncertain as a result of important cardiovascular disease in two cases and locally advanced synchronous bronchial carcinoma in two others.

\section{Patient Characteristics and Disease Manifestation}

Characteristics of the remaining 50 cases are shown in Table 1. Mean age was 63.7 years (range 32-85 years). Two of 15 prior airway cancers were synchronous bronchial cancer. Two other cases had synchronous colon carcinoma and papillary thyroid carcinoma, respectively.

Cases of ACC were younger (mean 57.9 years vs. 66.4 years, $P=.047)$, more often female $(61.5 \%$ vs. $37.0 \%$, NS), less often smokers $(58.3 \%$ vs. $91.7 \%$, $P=.017)$, and had fewer prior airway malignancies $(0 \%$ vs. $48.1 \%, P=.002)$ than those with SCC.

The four most common presenting symptoms were dyspnea, cough, hemoptysis, and stridor. Of 50 patients, in $47(94.0 \%)$ one or more, and in $36(72 \%)$ two or more of these symptoms were present. One tracheal carcinoma manifested as asymptomatic concomitant with bronchial cancer during workup of a nasal inverted papilloma. Ten patients were first seen at a tertiary center. Referral to tertiary centers occurred in 9 cases for treatment and in 18 
TABLE 1 Patient characteristics and referral pattern

\begin{tabular}{|c|c|c|}
\hline Characteristic & $n$ & $\%$ \\
\hline \multicolumn{3}{|l|}{ Sex } \\
\hline Male & 30 & 60 \\
\hline Female & 20 & 40 \\
\hline \multicolumn{3}{|l|}{ Comorbidities } \\
\hline Alcohol use & 15 & 30 \\
\hline Cardiovascular & 10 & 20 \\
\hline Chronic obstructive pulmonary disease & 8 & 16 \\
\hline Hypertension & 5 & 10 \\
\hline Diabetes & 5 & 10 \\
\hline Prior stroke & 2 & \\
\hline Pulmonary embolus & 2 & \\
\hline Deep vein thrombosis & 1 & \\
\hline Asthma & 1 & \\
\hline Obesity & 1 & \\
\hline Benign tracheal stenosis & 1 & 2 \\
\hline Other & 8 & 16 \\
\hline None & 19 & 38 \\
\hline \multicolumn{3}{|l|}{ Smoking history } \\
\hline Yes & 37 & 74 \\
\hline No & 7 & 14 \\
\hline Unknown & 6 & 12 \\
\hline \multicolumn{3}{|l|}{ Prior airway cancer } \\
\hline Lung & 11 & 22 \\
\hline Larynx & 3 & 6 \\
\hline Oropharynx & 1 & 2 \\
\hline \multicolumn{3}{|l|}{ Prior cancer, nonairway } \\
\hline Colon & 3 & 6 \\
\hline Prostate & 1 & 2 \\
\hline Breast & 1 & 2 \\
\hline Thyroid & 1 & 2 \\
\hline Uterine & 1 & 2 \\
\hline \multicolumn{3}{|l|}{ Referred to } \\
\hline Pulmonologist & 44 & 88 \\
\hline Internist & 3 & 6 \\
\hline Otolaryngologist & 2 & 4 \\
\hline Cardiologist & 1 & 2 \\
\hline \multicolumn{3}{|l|}{ Symptoms } \\
\hline Dyspnea & 33 & 66 \\
\hline Cough & 28 & 56 \\
\hline Hemoptysis & 21 & 42 \\
\hline Stridor & 18 & 36 \\
\hline Weight loss & 6 & 12 \\
\hline Hoarseness & 4 & 8 \\
\hline Dysphagia & 3 & 6 \\
\hline Neck mass & 3 & 6 \\
\hline Chest pain & 2 & 4 \\
\hline Fatigue & 2 & 4 \\
\hline Other & 3 & 6 \\
\hline
\end{tabular}

TABLE 1 continued

\begin{tabular}{|c|c|c|}
\hline Characteristic & $n$ & $\%$ \\
\hline None & 1 & 2 \\
\hline \multicolumn{3}{|c|}{ Duration of symptoms ${ }^{\mathrm{a}}$} \\
\hline$<1$ week & 2 & 4 \\
\hline $1-4$ weeks & 15 & 31 \\
\hline 1-3 months & 17 & 35 \\
\hline $3-6$ months & 9 & 18 \\
\hline 6-12 months & 2 & 4 \\
\hline$>12$ months & 4 & 8 \\
\hline \multicolumn{3}{|l|}{ Prior diagnosis $^{\mathrm{a}}$} \\
\hline Bronchitis & 7 & 14 \\
\hline Asthma & 4 & 8 \\
\hline Other & 3 & 6 \\
\hline None & 36 & 73 \\
\hline
\end{tabular}

${ }^{\text {a }}$ In 49 patients who had symptoms

for a second opinion, while 13 (26\%) were not referred. Median diagnostic delay was 9 days (mean 24 days) and ranged from 0 to 285 days.

\section{Diagnostic Procedures}

Diagnostic tests and tumor histology are summarized in Tables 2 and 3. Tumor and tracheal length were measured by endoscopy by the treating physician in $14(28 \%)$ of 50 cases. In six cases, cervical nodal invasion was found on CT and confirmed by biopsy. In five cases (10\%), chest CT showed pulmonary metastases. Abdominal CT revealed liver metastases in one case.

\section{Actual Treatment}

Primary treatment was radiotherapy in $29(58 \%)$ of 50 cases, combined with endobronchial treatment in 8, chemotherapy in 3, and both endobronchial treatment and chemotherapy in 5 . The radiation dose was $<39$ Gy in 6 cases, between 39 and $59 \mathrm{~Gy}$ in $4,>59 \mathrm{~Gy}$ in 19 , and $\geq 70$ Gy in 6 . Surgical resection was performed in 12 (24\%) of 50. Resection was preceded by endoscopic debulking in four cases. Postoperative adjuvant radiotherapy was administered at a dose of 39 to $59 \mathrm{~Gy}$ in four cases and $>59$ Gy in five. In 6 (12\%) of 50 cases, primary treatment consisted of endobronchial resection. In $3(6 \%)$ of 50 cases, only supportive care had been provided. Seven (54\%) of 13 patients with ACC underwent surgical airway resection.

\section{Radboud Panel Opinion}

In 10 cases without distant metastases, the tracheal tumor was considered unresectable because of mediastinal 
TABLE 2 Diagnostic and dissemination tests

\begin{tabular}{|c|c|c|}
\hline Test & $n$ & $\%$ \\
\hline \multicolumn{3}{|l|}{ Seen on chest X-ray } \\
\hline Yes & 14 & 28 \\
\hline No & 36 & 72 \\
\hline \multicolumn{3}{|l|}{ Seen on chest/neck CT } \\
\hline Yes & 42 & 84 \\
\hline No & 8 & 16 \\
\hline \multicolumn{3}{|l|}{ Endoscopic examination } \\
\hline Flexible & 17 & 34 \\
\hline Rigid & 5 & 10 \\
\hline Both flexible and rigid & 28 & 56 \\
\hline \multicolumn{3}{|l|}{ Seen at endoscopy } \\
\hline Yes & 50 & 100 \\
\hline No & 0 & 0 \\
\hline \multicolumn{3}{|l|}{ Localization } \\
\hline Proximal & 19 & 38 \\
\hline Middle & 11 & 22 \\
\hline Distal & 20 & 40 \\
\hline \multicolumn{3}{|l|}{ Dissemination tests } \\
\hline CT liver and adrenal glands & 29 & 58 \\
\hline PET scan & 14 & 28 \\
\hline Scintigraphy & 6 & 12 \\
\hline Esophagoscopy & 6 & 12 \\
\hline US abdomen & 5 & 10 \\
\hline Histology neck node & 5 & 10 \\
\hline MRI of the chest & 4 & 8 \\
\hline US neck & 4 & 8 \\
\hline CT or MRI brain & 3 & 6 \\
\hline Endoesophageal US & 3 & 6 \\
\hline Mediastinoscopy & 2 & 4 \\
\hline High-resolution chest $\mathrm{CT}$ & 2 & 4 \\
\hline Swallow X-ray & 1 & \\
\hline
\end{tabular}

$C T$ computed tomography, PET positron emission tomography, US ultrasound, MRI magnetic resonance imaging

invasion in 5, excessive length in 4 , and dissemination during previous thyroidectomy in 1 . The panel regarded three cases as resectable but inoperable as a result of advanced age or local recurrence of a peripheral lung cancer. In $31(62 \%)$ of 50 primary tracheal carcinoma, the panel advised surgical tracheal resection with curative intent.

\section{Opinion of Second Reviewer}

In $4(8 \%)$ of 50 cases, the individual thoracic surgeon differed from the panel regarding tumor resectability. The second reviewer judged three cases considered resectable by the panel as unresectable because of prior cervicomediastinal radiation. The Radboud panel considered one case
TABLE 3 Histological types found in 50 cases of primary tracheal carcinoma

\begin{tabular}{lrr}
\hline Disease & $n$ & $\%$ \\
\hline SCC & 27 & 54 \\
ACC & 13 & 26 \\
Carcinoid & 4 & 8 \\
Large cell carcinoma & 3 & 6 \\
Adenocarcinoma & 2 & 4 \\
Small cell carcinoma & 1 & 2 \\
\hline
\end{tabular}

$S C C$ squamous cell carcinoma, $A C C$ adenoid cystic carcinoma

judged resectable by the second reviewer as unresectable as a result of lymphadenopathy. Thus, there were 28 candidates for resection as determined by both the Radboud panel and the second reviewer.

\section{Potential Surgical Candidates}

Of 28 potential surgical candidates, actual treatment in 16 cases $(57 \%)$ consisted of other modalities: radiotherapy in 11, endobronchial therapy in 4, and supportive care in 1. Only $12(43 \%)$ of 28 of surgical candidates actually underwent surgical resection. Age, histological type, and tumor length in these groups are listed in Table 4. Contraindications to surgery that were stated in the medical chart are listed in Table 5. Surgical consultation had been obtained in half ( 8 of 16) of surgical candidates who had not undergone resection.

If we assume that none of the 17 cases excluded from review as a result of insufficient clinical information would have been a candidate for tracheal resection, $28(42 \%)$ of 67 of cases would have been surgical candidates, as opposed to the actual resection rate of $12(18 \%)$ of 67 $(P=.003)$.

\section{DISCUSSION}

An audit review of the NCR found metastases to the trachea in one-third of cases registered, and therefore in our view misclassified as primary tracheal carcinoma. Our study further finds that fewer than half of all patients with resectable tumors undergo surgical resection. These findings highlight the limited validity of epidemiologic data for this and other rare diseases that are reported to the registry without radiologic or histologic review. More importantly, the audit points to problems in the clinical care of patients with uncommon tracheal tumors.

The concept of unsolicited panel and radiographic review of all registered cases of a certain type of tumor in one country in a designated period is unique in its design and to our knowledge has never been described before. 
TABLE 4 Patient and tumor characteristics in surgical and nonsurgical candidates

\begin{tabular}{|c|c|c|c|c|c|c|c|c|c|}
\hline \multirow[t]{2}{*}{ Candidates } & \multirow[t]{2}{*}{$N$} & \multicolumn{2}{|c|}{ Age (year) } & \multicolumn{4}{|c|}{ Histological type (\%) } & \multicolumn{2}{|c|}{ Tumor length $(\mathrm{cm})$} \\
\hline & & Mean & Range & $\mathrm{SCC}$ & $\mathrm{ACC}$ & $\mathrm{Cd}$ & Other & Mean & Range \\
\hline Surgical candidates & 28 & $61.4^{\mathrm{a}}$ & $32-80$ & 32.1 & 35.7 & 14.3 & 17.9 & $3.0^{\mathrm{b}}$ & $1.0-5.5$ \\
\hline Resected & 12 & $57.8^{\mathrm{c}}$ & $37-75$ & 33.3 & 58.3 & 8.3 & 0 & $2.8^{\mathrm{d}}$ & $1.5-5.5$ \\
\hline Not resected & 16 & $64.3^{\mathrm{c}}$ & $32-80$ & 31.3 & 18.8 & 18.8 & 31.3 & $3.3^{\mathrm{d}}$ & $1.0-5.2$ \\
\hline Nonsurgical candidates & 22 & $66.5^{\mathrm{a}}$ & $46-85$ & 81.8 & 13.6 & 0 & 4.5 & $5.5^{\mathrm{b}}$ & $1.4-12.0$ \\
\hline Overall & 50 & 63.7 & $32-85$ & 54.0 & 26.0 & 8.0 & 12.0 & 4.2 & $1.0-12.0$ \\
\hline
\end{tabular}

$S C C$ squamous cell carcinoma, $A C C$ adenoid cystic carcinoma, $C d$ carcinoid

${ }^{\mathrm{a}} P=.165$

b $P<.0001$

${ }^{c} P=.190$

${ }^{\mathrm{d}} P=.358$

TABLE 5 Documented reasons for nonsurgical management in 16 nonresected surgical candidates

\begin{tabular}{llr}
\hline Reason & $n$ & $\%$ \\
\hline Tracheal process unresectable & 7 & 43.8 \\
$\quad$ Segment too long & 2 & 12.5 \\
$\quad$ Possible ingrowth muscular esophagus & 1 & 6.3 \\
$\quad$ Long dysplastic area in trachea & 1 & 6.3 \\
$\quad$ Not documented & 1 & 6.3 \\
Carcinoid tumor & 3 & 18.8 \\
Patient preference & 1 & 6.3 \\
Not documented & 7 & 43.8 \\
\hline
\end{tabular}

Although external auditing of blinded and abstracted data by a panel of experts itself is subject to limitations, this method approximates the concept of multidisciplinary oncologic review by local experts adopted for common tumors in everyday practice. Treatment advice of our panel review is furthermore reproducible: the interreviewer disagreement between the Radboud panel and the second reviewer was small (8\%). In rare diseases such as tracheal tumors, however, the pool of experts is small and often not local. The emphasis on local surgical assessment and care may imply a potentially profound difference in the indications for surgical therapy and the outcome of individual patients.

The high proportion of misclassified tracheal carcinomas in the NCR suggests that the registration process for uncommon tumors may be improved. Errors in this classification consisted of a misattribution of tumors originating from adjacent organs or mediastinal lymphadenopathy with invasion of the airway wall to the trachea. Results of any biopsy of tumor from the trachea are reported to the NCR as tracheal cancer through the direct link with the national pathology database (PALGA). The final registration in the NCR database, however, involves a review of the patient medical chart. Some of the misdiagnoses may therefore have occurred when the tumor site of origin was misclassified in the report to the NCR. A retrospective review may correct the database error, but not the implications for patient care.

Three other epidemiologic studies of national cancer registry data also found misclassified tracheal carcinomas. A Finnish study found 36 metastatic tracheal lesions among 133 registered primary carcinomas $(27.1 \%)$, while a Danish audit excluded 16 misclassified tumors from 130 registered cases $(12.3 \%))^{7,10}$ In a review of the American Surveillance, Epidemiology, and End Results (SEER) database, Bhattacharyya disregarded cases with distant metastasis and excluded an additional 7 (7.1\%) of 99 cases with carcinoma-in-situ or unclear pathology. ${ }^{11}$ None of these studies, however, included a complete radiological review and thus may have underestimated the proportion of misclassified metastatic tumors. Conversely, misclassification could also have occurred by labeling cases of primary tracheal cancer as metastatic bronchial, laryngeal, esophageal, or thyroidal carcinoma. This error, however, is expected to occur less frequently because the registry is linked to the site of biopsy, requiring active intervention by the responsible physician to change the tumor site.

A critical assessment of our previous study on incidence and treatment of tracheal carcinoma, also on the basis of the files of the NCR but without review of radiographs, now suggests that the incidence of this disease in the Netherlands is overestimated and probably closer to .1 in every 100,000 persons per year. ${ }^{6}$ The high rate of small cell carcinoma in our previous study $(11.0 \%)$, regarded as a marker of contamination with metastatic disease, was likely caused by the misclassification of peripheral bronchial carcinomas. ${ }^{12}$ We estimate that ACC accounts for approximately one-quarter of tracheal cancers. In surgical series, ACC is encountered in $40 \%$ to $60 \%$ of cases. ${ }^{13-15}$ The reported incidence of SCC lies fairly constant at 
approximately $50 \%$ in both epidemiologic and surgical series. $^{3,6,16-18}$ Future epidemiologic studies of tracheal carcinoma and other tumors of low incidence should therefore include an audit review of radiographic and, where feasible, also histologic images.

Our study had important limitations. A retrospective case review does not include patient contact, physical assessment, or endoscopic examination. The locoregional extent of disease, in the context of patient factors such as age, weight, and neck mobility, is the single most important factor determining resectability. ${ }^{2}$ Despite panel review of CTs, endoscopy reports were usually brief and often nondescriptive. Endoscopic measurements of tracheal and tumor length were missing in $72 \%$ of patients. Thus, the actual resectability rate may be lower than our estimate. Still, tumor length in potential surgical candidates was well within the range of resectable disease, and differences of mean and range of tumor length between patients treated with resection and additional surgical candidates were small (mean 2.8 and $3.3 \mathrm{~cm}$, respectively). ${ }^{2} \mathrm{~A}$ further potential source of error in our study was the determination of operability status based on available evidence in the patient's medical chart. However, the Radboud panel excluded four cases in which the absence or presence of medical contraindications to surgery could not be determined, even though patients' disease is rarely declared unresectable as a result of medical contraindications. ${ }^{2}$ As depicted in Table 5, no medical contraindications were identified in any of the 16 additional surgical candidates who did not undergo surgical resection.

Our results provide additional evidence that half of all patients with primary tracheal cancer are surgical candidates. ${ }^{4,5}$ The balance is tipped furthest toward resection in ACC: the Radboud panel judged 10 (77\%) of 13 cases to have resectable disease. Because this national audit was preceded by epidemiologic studies in Denmark, Finland, and the United Kingdom with comparable outcome with those in the Netherlands, these results are likely representative for the situation in most western European countries. ${ }^{6-8,16}$

We believe that each patient diagnosed with a tracheal tumor should be referred to a tertiary oncology center with multidisciplinary experience in the treatment of tracheal tumors. Given the rarity of the disease, to warrant sufficient exposure to malignant tracheal pathology and to maintain experience, one center per each population of an estimated 10 to 20 million would be optimal. In the Dutch situation, this would mean one national center. By centralizing the care for patients with this rare airway tumor, more patients may be selected for surgical resection, thus potentially improving outcome. Because this improvement is inferred but untested, close evaluation would be needed once a centralized system is implemented to assess the exact benefit of this new strategy.
ACKNOWLEDGMENTS None of the authors had nor currently has any financial or personal relationships with other people or organizations that could inappropriately influence or bias their work. There are no conflicts of interest. There were no sources of funding other than departmental funds. The corresponding author (J.H.) had full access to all the data in the study and had final responsibility for the decision to submit for publication. We thank all involved physicians (pulmonary physicians, otolaryngologists, surgeons, radiotherapists, internists, cardiologists, neurologists) and their secretaries for the collection of the patient-related data for their cooperation in this study. We thank the personnel of the nine regional cancer registries in the Netherlands for their help in identifying patients with tracheal cancer, obtaining permission from the physicians, and collecting patient charts. We thank all involved archives of radiology for the availability of conventional and digital radiological images, and we thank all involved medical archives for the availability of patient charts.

Open Access This article is distributed under the terms of the Creative Commons Attribution Noncommercial License which permits any noncommercial use, distribution, and reproduction in any medium, provided the original author(s) and source are credited.

\section{REFERENCES}

1. Macchiarini P. Primary tracheal tumours. Lancet Oncol. 2006; 7:83-91.

2. Gaissert HA, Grillo HC, Shadmehr MB, et al. Long-term survival after resection of primary adenoid cystic and squamous cell carcinoma of the trachea and carina. Ann Thorac Surg. 2004; 78:1889-97.

3. Regnard JF, Fourquier P, Levasseur P. Results and prognostic factors in resections of primary tracheal tumors: a multicenter retrospective study. The French Society of Cardiovascular Surgery. J Thorac Cardiovasc Surg. 1996;111:808-13.

4. Hazama K, Miyoshi S, Akashi A, et al. Clinicopathological investigation of 20 cases of primary tracheal cancer. Eur J Cardiothorac Surg. 2003;23:1-5.

5. Gaissert HA. Primary tracheal tumors. Chest Surg Clin N Am. 2003;13:247-56.

6. Honings J, van Dijck JA, Verhagen AF, et al. Incidence and treatment of tracheal cancer: a nationwide study in the Netherlands. Ann Surg Oncol. 2007;14:968-76.

7. Licht PB, Friis S, Pettersson G. Tracheal cancer in Denmark: a nationwide study. Eur J Cardiothorac Surg. 2001;19:339-45.

8. Manninen MP, Pukander JS, Flander MK, et al. Treatment of primary tracheal carcinoma in Finland in 1967-1985. Acta Oncol. 1993;32:277-82.

9. Fritz A, Percy C, Jack A, et al. International classification of diseases for oncology. 3rd ed. Geneva: World Health Organization, 2000.

10. Manninen MP, Antila PJ, Pukander JS, et al. Occurrence of tracheal carcinoma in Finland. Acta Otolaryngol. 1991;111:1162-9.

11. Bhattacharyya N. Contemporary staging and prognosis for primary tracheal malignancies: a population-based analysis. Otolaryngol Head Neck Surg. 2004;131:639-42.

12. Grillo HC. Primary tracheal tumours. Thorax. 1993;48:681-2.

13. Pearson FG, Todd TR, Cooper JD. Experience with primary neoplasms of the trachea and carina. J Thorac Cardiovasc Surg. 1984;88:511-8.

14. Perelman MI, Koroleva N, Birjukov J, et al. Primary tracheal tumors. Semin Thorac Cardiovasc Surg. 1996;8:400-2.

15. Grillo HC, Mathisen DJ. Primary tracheal tumors: treatment and results. Ann Thorac Surg. 1990;49:69-77. 
16. Gelder CM, Hetzel MR. Primary tracheal tumours: a national survey. Thorax. 1993;48:688-92.

17. Kurien G, Cole I. Primary carcinoma of the trachea. Clin Otolaryngol. 1981;6:197-204.
18. Yang KY, Chen YM, Huang MH, et al. Revisit of primary malignant neoplasms of the trachea: clinical characteristics and survival analysis. Jpn J Clin Oncol. 1997;27:305-9. 